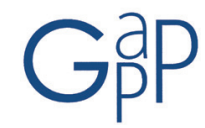

GESTIÓN Y ANÁLISIS DE POLÍTICAS PÚBLICAS, Nueva Época, nº 13 enero-junio 2015 ISSN: 1989-8991

DOI: http://dx.doi.org/10.24965/gapp.voi13.10230

\title{
El acceso a la información pública y su impacto en la corrupción. El caso de
} México

\author{
Martín Vera Martínez \\ Universidad Autónoma de Baja California, México \\ martin.vera@uabc.edu.mx
}

\begin{abstract}
Resumen
El presente artículo tiene como fin presentar un análisis, sobre la base de un estudio de caso que permita corroborar aspectos ya expuestos por otros autores respecto de la relación entre transparencia y corrupción. A tal fin se analizará el caso mexicano y se sostendrá que los cambios normativos que facilitan el acceso a la información no son suficientes para influenciar positivamente en la relación entre transparencia y corrupción que, debido a su complejidad, debe contemplar otras variables para contribuir efectivamente en el contexto señalado.
\end{abstract}

\section{Palabras clave}

Transparencia, acceso a la información pública, corrupción, México, participación ciudadana.

\section{Access to public information and its impact on corruption. The case of Mexico}

\section{Abstract}

This paper aims to present a analysis, based on a case study to corroborate aspects already discussed by other authors regarding the relationship between transparency and corruption. To this end the Mexican case is analyzed and it will be argued that normative reforms which facilitate the access to information are not enough to positively influence the relationship between transparency and corruption which, due to its complexity, should consider other variables in order to really contribute the context indicated.

\section{Key words}

Transparency, public information access, corruption, México, citizen participation. 


\section{INTRODUCCIÓN}

Desde los tiempos de Aristóteles y su relato sobre La Política se ha destacado la importancia de la evolución de la democracia en un marco de libertad, en donde los ciudadanos puedan tener acceso a juzgar los asuntos públicos con el fin de generar un equilibrio de las fuerzas políticas. La información, como punto angular de un sistema democrático ha conllevado a la participación ciudadana en los actos de gobierno y ha sido abordada desde la democracia ateniense hasta nuestra actualidad. Sin embargo, el desarrollo de la vida política no ha sido ajena al mal uso del poder público para conseguir una ventaja ilegítima por medio de actos de corrupción que han tratado de ser combatidos por medio de reformas jurídicas, resoluciones gubernamentales y diseño de políticas públicas, en las que la principal estrategia para disminuir la corrupción en el Estado ha sido concentrado en ampliar y facilitar las vías de acceso del ciudadano a la información pública relativa a los actos de gobiernos. De esta manera, se presenta una relación compleja entre transparencia y corrupción donde el acceso a la información no resulta en determinados casos suficiente para contribuir positivamente a dicha relación de naturaleza compleja, debiéndose complementar con otros factores.

El presente artículo tiene como fin analizar un estudio de caso que permita corroborar aspectos ya expuestos por otros autores respecto de la relación entre transparencia y corrupción. A tal fin se analizará el caso mexicano y se sostendrá que los cambios normativos que facilitan el acceso a la información no son suficientes para influenciar positivamente en la relación entre transparencia y corrupción que, debido a su complejidad, debe contemplar otras variables para contribuir positivamente en el contexto señalado. En tal sentido se presentará en primer lugar una revisión de la literatura sobre los ejes temáticos de estudio, a saber la corrupción, la transparencia y la naturaleza de la relación entre ellos. En segundo lugar, se expondrán los criterios metodológicos En tercer lugar se analizarán los datos presentados para finalmente presentar las conclusiones de la investigación.

\section{REVISION DE LA LITERATURA. TRANSPARENCIA DE LA INFORMACIÓN PÚBLICA Y EL FENÓMENO DE LA CORRUPCIÓN EN LOS ACTOS DE GOBIERNO. RELACIÓN ENTRE TRANSPARENCIA Y CORRUPCIÓN}

La transparencia de la información pública se ve motivada a ejercerse o demandarse por tener el atractivo de abatir la corrupción, optimizar la eficiencia de las instancias gubernamentales y mejorar la calidad de vida de las personas al tener éstas la posibilidad de utilizar las leyes de acceso y transparencia como herramienta para nutrir de mejores contenidos la toma cotidiana de decisiones que se realizan día con día. En este orden de ideas, cuando la transparencia se encuentra orientada hacia el ejercicio o administración pública, hace referencia a valores sociales como la honestidad, la claridad, la participación y el respeto a las reglas tanto para el gobernante como para el gobernado (Bellver, 2007). Si bien no existe una definición uniforme de la transparencia, lo cierto es que tanto los conceptos vertidos por los organismos internacionales y la doctrina especializada en la materia son coincidentes respecto de que la definición de transparencia se encuentra estrechamente relacionada con la confianza. Según Hilda Nassens (2010), la transparencia "es un atributo o cualidad que permite tener más información clara y precisa sobre una persona o algo, lo que redundaría en el aumento de nuestras capacidades de comprensión, vigilancia y comunicación". Conforme a lo indicado, podemos definir a la transparencia como la demanda de los ciudadanos hacia los mercados y a los gobiernos para sostener instituciones confiables tanto por sus políticas como por sus resultados (Secretaría de Gobernación, 2013). La referencia a la calidad de la confiabilidad es destacada por Florini (1999), quien aporta su visión al ejercicio de la transparencia por medio de actos tales como la "la divulgación, por parte de las instituciones, de información que es relevante para evaluarlas". Así entendida, la transparencia permite la divulgación de información sobre las instituciones que son relevantes para la evaluación de los organismos públicos y, en tal sentido, es un tema que ha generado gran preocupación para las ciencias sociales contemporáneas, al grado de reconocer su contribución a la búsqueda de un régimen de administración eficaz y democrática. Por supuesto que tal conceptualización no es ajena a su rol frente a la administración pública, ya que transparentar la gestión pública, como sostiene Ontiveros (2005), implica favorecer la rendición de cuentas a los ciudadanos ejerciendo su derecho humano a demandar información precisa, confiable y comprobable de su mandato al gobierno.

La transparencia puede dividirse en dos grandes grupos de ejecución de la política de acceso a la información. Por un lado, existen aquellas políticas llamadas también de primera generación, que apuntan a abrir los archivos oficiales o de acceso a la información pública y su expresión más conocida la constituyen las leyes de acceso a la información (Molina \& Vieyra, 2012). Dichas políticas proponen una dinámica verticalista que sostiene que si se tiene acceso a la información pública, los ciudadanos contarán con las herramientas fundamentales para controlar mejor lo que hacen los gobiernos, fortalecer la participación ciudadana y la democracia moderna (Rose-Ackerman, 1999). Cabe destacar que las leyes de acceso a la información, como ejemplo de políticas de transparencia de primera generación, tienen generalmente su origen en contextos de recambio político a nivel nacional; reflejan 
objetivos amplios y abstractos, y no necesariamente los ciudadanos entienden que estén relacionadas con su vida cotidiana (Vieyra, 2009). Ejemplo de lo antedicho son las estrategias anticorrupción, los planes de acción y la creación de organismos y comisiones especializadas en la lucha contra la corrupción, lo cual no implica que para la realización de determinado objetivo se emprendan políticas de segunda generación o bien éstas últimas combinadas con las de primera.

Por otra parte, el otro grupo de políticas de transparencia, denominadas de segunda generación, o transparencia focalizada, tienen como objetivo "la divulgación de una información específica, identificando y diagnosticando un objetivo político preciso para la divulgación de información” (Dassen et al., 2012), generalmente pudiendo obtener resultados concretos en el corto y el mediano plazos respecto del comportamiento esperado de las entidades objetivo, según el nivel de la comprensión y coordinación de los usuarios de la información. Sin embargo, cabe destacar que dicha información, aunque pueda ser disponible y accesible, al no ser comprendida por quien la accede puede tener como resultado el desinformar o bien diferir de la que se espera obtener a través de una política de acceso a la información a nivel general (Graham, 2002). De esta manera, la transparencia como concepto constituye una exigencia ubicua en el mundo de la política democrática: obliga al poder a abrirse a la inspección pública; lo fuerza a explicar y justificar sus actos y lo supedita a la amenaza de sanciones. Involucra por tanto el derecho a recibir información y la obligación correspondiente de divulgar todos los datos necesarios, recibir una explicación y el deber correspondiente de justificar el ejercicio del poder. El funcionamiento de las instituciones del Estado, acompañadas de un ejercicio eficaz, eficiente y ético de la gestión pública constituye el sustento de cualquier sociedad que quiera consolidarse como democrática.

Con relación al fenómeno de la corrupción -derivado del latín corruptio, y cuyo significado tiene distintas acepciones como son descomposición, putrefacción, degradación, depravación, perversión y malignidad-, se conoce que es Aristóteles el primero en utilizar este vocablo, hoy tan extendido y multicultural, mismo que invoca para referirse a la degeneración que alcanzan las formas de gobierno monárquico, aristocrático y democrático, derivándose de su génesis las formas corruptas que son respectivamente, tiranía, oligarquía y demagogia. De forma más actualizada, Della Porta y Vanucci (2002) la definen como "aquellas acciones u omisiones que tienen que ver con el uso abusivo de los recursos públicos para beneficios privados". Es decir, estaremos en presencia de un acto de corrupción cuando se produzca una violación de alguna norma -ya sea por acción u omisión- establecida por funcionarios o actores sociales encargados de vigilar, gestionar o administrar los recursos públicos. Dentro de las principales características del fenómeno podemos destacar el rompimiento del tejido social, puesto que su presencia disminuye la confianza de los ciudadanos en las instituciones, el gobierno y entre ellos mismos, afectando el nivel ético de la sociedad en su conjunto y, como fenómeno mundial, todos los tipos de gobierno son susceptibles en alguna medida de su existencia. Stephen D. Morris (1991) elaboró tres proposiciones básicas para explicar el origen de la corrupción, siendo destacable para nuestro análisis aquel que reza que la corrupción tendrá origen cuando exista un desequilibrio estructural que influye sobre las organizaciones sociales para influir sobre el comportamiento político.

De esta manera y tomando en consideración lo referido, la relación entre transparencia y corrupción ha sido ya señalada en la doctrina. Investigaciones previas realizadas por Álvaro Cuervo Cazurra (2014) concluyeron que la transparencia por sí misma no es suficiente para combatir la corrupción, y que la misma debe ser complementada por sistemas de control y castigo para los agentes que cometen actos de corrupción, incluyendo un rol activo de sociedad civil y los medios de comunicación. En este sentido, Bauhr y Grimes (2014) indican que la relación entre transparencia y corrupción no es sincrónica y predecible, sino que por el contrario debe ser examinada a fin de poder considerar otros factores que la complementen para poder generar impactos positivos en la reducción de los niveles de corrupción, ya que no siempre niveles altos de transparencia se traducen en una disminución del fenómeno de la corrupción. Lindstedt y Naurin (2010) también argumentan que no necesariamente instituciones políticas más transparentes sean un método efectivo para combatir la corrupción, donde la publicidad y la rendición de cuentas por sí mismas no serían la solución al problema. La tesis de los autores concluye que soluciones externas generadas fuera del sistema institucional pueden ser más efectivas y complementarias a la relación transparencia-corrupción, como sería la generación de un entorno que promueva la libertad de prensa. En palabras de Aguilar-Bustelo (2010) el ejercicio estándar de evaluación -de la relación transparencia/corrupción- no cuestiona la estructura de la política y del programa sino que la presupone correcta. En este sentido, Arellano Gault (2008) sostiene que la transparencia como criterio establece una serie de parámetros deseables en una interacción organizativa que son difíciles de alcanzar a menos que exista un esfuerzo específico de los actores por incorporarla e internalizarla. Es por ello que las organizaciones deben construir la transparencia y no sólo adaptarla o localizarla. Podemos concluir entonces que la literatura confronta la idea que admite como verdadera la relación causal esta- 
blecida entre transparencia y corrupción, esto es, que a mayor transparencia del ejercicio de funciones y a mayor acceso a la información pública, menor grado de corrupción vigente en una sociedad determinada, sino que propicia la indagación de otros complementos o factores que deban ser insertados en la relación entre transparencia y corrupción.

\section{HIPÓTESIS Y METODOLOGÍA}

Tal como lo evidencia la literatura, existe una relación entre la transparencia de los actos públicos -y en consecuencia el acceso a la información por parte de los ciudadanos- y los niveles de corrupción de un Estado. Ahora bien, si el Estado Mexicano cuenta con mecanismos normativos tendientes a facilitar políticas de transparencia para un mayor acceso a la información por parte de los ciudadanos, ¿son dichas normas suficientes para impactar positivamente en la relación entre transparencia y corrupción en tal contexto? ¿El caso de México obedece a la relación entre transparencia y corrupción? ¿Han impactado las políticas de gobierno abierto en la disminución de los casos de corrupción? ¿El acceso a la información pública necesita de otros mecanismos para disminuir los índices de corrupción? Sostenemos que en el caso mexicano existe una relación entre transparencia y corrupción y que la misma no refleja, hasta el momento, indicadores positivos respecto de la disminución de la corrupción en el Estado.

A fin de poder corroborar la hipótesis señalada, se analizarán dos tipo de fuentes, por un lado fuentes normativas y por el otro el indicador de percepción de la corrupción (IPC) ${ }^{1}$ de la organización Transparency International $(\mathrm{TI})^{2}$. Respecto de las fuentes normativas, se presentarán las principales normas jurídicas que permiten al ciudadano ejercer su derecho de acceso a la información pública y exigir la transparencia en los actos de gobierno. Con relación al indicador de $\mathrm{Tl}$, se ha decidido utilizarlo debido a que ha sido reconocido por más de 90 países en el mundo como indicador certero respecto del grado de percepción de la corrupción por parte de los ciudadanos de los países que se auditan. Asimismo, la labor de TI ha sido reconocida por organizaciones internacionales tales como la Organización para la Cooperación y el Desarrollo Económicos, el Banco Mundial, el Foro Económico Mundial y por cada uno de los países de la organización, incluido México. El marco temporal elegido para la selección de las fuentes será desde 1998 hasta 2013.

Luego de presentar el relevamiento de las fuentes, se ofrecerá la relación entre transparencia y corrupción por medio de la comparación de los resultados. Así, se compararán las variables para poder indagar si existe una relación "más-menos" entre los mecanismos normativos y el índice de corrupción. Con mayor precisión, se tratará de examinar si hay una relación proporcional que refleje que a mayor número de normas que se han generado para posibilitar la transparencia de los actos públicos existe una mayor disminución del índice de corrupción. Por el contrario, si lo antedicho no se evidencia, analizaremos qué otros factores debieran tenerse en consideración para complementar dicha relación e impactar positivamente en la disminución de la corrupción en el caso mexicano. Cabe aclarar que si bien no desconocemos que existen determinadas normas que para tener incidencia en los índices de corrupción debieran cotejar un marco temporal más extenso, consideramos que el presente trabajo tiene como fin realizar un aporte sobre la situación actual de la relación transparencia-corrupción en México, dejando abierta la exploración temporal y el impacto de las últimas reformas administrativas para próximas investigaciones.

\section{RESULTADOS DEL RELEVAMIENTO DE DATOS. VARIABLES NORMATIVAS Y ESTADÍSTICAS}

\section{El sistema de acceso a la información pública y transparencia en México (1998-2013)}

Las reformas normativas registradas entre 1998 a 2013 para crear una cultura de la transparencia y de rendición de cuentas se inscriben en tres grandes vertientes: el robustecimiento del control interno cada uno de los tres poderes federales, la neutralización del sistema (los tres poderes empiezan a vigilarse mutuamente) y el avance en la capacidad de la ciudadanía para acceder a la información generada por el gobierno, en concordancia con procesos similares que se replican internacionalmente. En México, el derecho a la información se incluyó por primera

1 Dicho indicador es una "encuesta de encuestas" basadas en información de diversas encuestas de opinión pública (como el Banco Mundial, la Universidad de Columbia o el Foro Económico Mundial) sobre la magnitud de la corrupción en 120 países, que refleja las percepciones de empresarios, analistas de riesgo, especialistas financieros y otros, de la situación de la corrupción en cada país. Además TI ha instaurado capítulos nacionales que desarrollan diversas actividades de monitoreo, evaluación de políticas nacionales y procesos gubernamentales.

2 Al nivel internacional, el movimiento TI tiene como objetivo principal infundir al sistema global de valores la transparencia y la rendición de cuentas como normas públicamente reconocidas. La Secretaría Internacional de TI trabaja con el sector privado y organizaciones internacionales tales como la OCDE, para fortalecer el marco político y legal de los negocios internacionales. Mientras la Secretaría Internacional dirige la agenda internacional de la organización, más de 80 Capítulos Nacionales en distintos países encabezan el movimiento de TI a nivel de base. Para mayor información consultar http://www.transparency.org/ 
vez en la Constitución de 1997, en el marco de la denominada "reforma política", en donde se modificó el Artículo $6^{\circ}$ constitucional para añadir el texto que reza el derecho a la información será garantizado por el Estado, un precepto complementario del Artículo $8^{\circ}$ que ya reconocía el derecho de petición3. Tal como lo señala el Dictamen de la Cámara de Senadores, "mediante la reforma..., se ha elevado a rango constitucional el derecho de la información, que es una de las bases de sustentación de la democracia como sistema de vida" 4.5 Sin embargo tal garantía individual careció de soporte en la legislación secundaria hasta la promulgación de la Ley Federal de Transparencia y Acceso a la Información Pública Gubernamental (LFTAIPG), publicada en el Diario Oficial de la Federación (DOF) el 11 de junio de 20026. Además se creó el Instituto Federal de Acceso a la Información Pública, como un órgano de la Administración Pública Federal, con autonomía operativa, presupuestaria y de decisión, cuyo objetivo radicó en promover y difundir el ejercicio del derecho de acceso a la información, resolver la negativa a las solicitudes de acceso a la información y proteger los datos personales en posesión de las dependencias y entidades de la Administración Pública Federal.

El 5 de julio de 2010 se publicó en el DOF un decreto por el que se reformaron los Artículos $3^{\circ}$ (fracciones II y VII), y $33^{\circ}$ de la LFTAIPG, lo que implicó la modificación de la denominación del "Instituto Federal de Acceso a la Información” por la de “Instituto Federal de Acceso a la Información y Protección de Datos”. A la ley le siguieron otras leyes equivalentes en las entidades federativas. Sin embargo, éstas no fueron una mera copia de la ley federal, sino que siguieron diferentes modelos. Para finales de 2007 todos los estados de la república contaban con una ley en la materia aunque los criterios y normas de esas leyes variaban notablemente. De igual forma en este marco histórico se enunciaron los propósitos del Plan Nacional de Desarrollo 2001-2006 que tenían como objetivo construir un gobierno abierto y transparente, que trabaje en una gran vitrina de cristal en donde todos los ciudadanos sepan el porqué de sus acciones y cuenten con acceso permanente a la información.

En respuesta a este problema, se gestó un movimiento que pugnaba por una reforma constitucional que permitiera eliminar las diferencias en el ejercicio del mismo derecho. Un grupo de gobernadores presentó a la consideración del Congreso de la Unión un documento conocido como la "Iniciativa Chihuaha", en donde se proponía añadir al Artículo $6^{\circ}$ constitucional los criterios mínimos para ejercer el derecho de acceso a la información. Este documento fue la base para que el Constituyente Permanente aprobara en 2007 la reforma que adicionó el segundo párrafo del Artículo $6^{\circ}$. El texto reformado de dicho Artículo contiene los principios y bases del ejercicio del derecho en todo el país; en otras palabras, establece el estándar mínimo del ejercicio del derecho y sienta los principios de la política de transparencia gubernamental,7 lo que significa que debe facilitarse el desarrollo de mecanismos de acceso que permitan a cualquier persona realizar y obtener de manera expedita el acceso a la información, a sus datos personales y a la rectificación de estos últimos. Luego de la reforma, se reformaron 19 leyes y se expidieron 12 nuevas. En materia federal, el Senado de la República aprobó en diciembre de 2009 una reforma sustantiva a la LFTAIPG. Primeramente se crearon órganos garantes de acceso a la información, los cuales tienen como función central sustanciar de manera expedita los procedimientos de revisión respecto de las negativas de acceso a la información. En segundo lugar, se reconoce el derecho a la protección de los datos personales y los derechos conexos de acceso, rectificación, cancelación y oposición. ${ }^{8}$ La última reforma se llevó acabo el 7 de

3 Si bien es cierto que durante muchos años existió el derecho de petición, reconocido por el Artículo $8^{\circ}$ de la Constitución, éste sólo obligaba a la autoridad a emitir acuerdo por escrito con respecto a una solicitud, también presentada por escrito por un particular. Tal acuerdo podía negar una solicitud de información, argumentando que era un “secreto administrativo", o que la autoridad no la tenía, o que carecía de facultades para divulgarla. La ausencia de respuesta, por regla general, se consideraba una negativa ficta.

4 Cámara de Diputados, LV Legislatura, Derechos del pueblo mexicano, México a través de sus Constituciones, México: Porrúa, p.372.

5 En un primer momento, se entendió que este derecho se concretaba al garantizar el acceso de los partidos políticos a los medios de comunicación, posteriormente su interpretación se centró en el debate sobre las implicaciones que tendría tal derecho sobre la regulación de los medios de comunicación en el país. Finalmente su concepción fue ampliándose considerando tal derecho como una "garantía” que implicaba el acceso a la información gubernamental

6 Para la elaboración de la ley fueron tomadas en cuenta las iniciativas del diputado Miguel Barbosa; la del presidente Vicente Fox y los grupos parlamentarios del PRD, PRI, PT, PVEM y Convergencia Democrática, elaborada por el Grupo Oaxaca. Ver Escobedo, Juan Francisco (2003). Movilización de opinión pública en México: el caso del Grupo Oaxaca y de la Ley Federal de Acceso a la Información Pública, Derecho comparado de la información, México: Instituto de Investigaciones Jurídicas. También se hizo referencia a la emisión del Código de Ética para la Administración Pública Federal, el cual fue publicado en el Diario Oficial de la Federación el 31 de julio del año 2002. Este Código tiene como propósito la difusión de reglas claras para que, en la actuación de los servidores públicos, impere invariablemente una conducta digna.

7 Para un análisis detallado del contenido de la reforma constitucional véase, entre otros, Bustillos y Carbonell (2007). Hacia una democracia de contenidos: la reforma constitucional en materia de transparencia. México: Instituto de Investigaciones Jurídicas de la Universidad Autónoma de México. Carbonell, Miguel, 2008, El régimen constitucional de la transparencia. México: UNAM; Salazar, Pedro (2008). El derecho de acceso a la información en la Constitución Mexicana. Razones, significados y consecuencias. México: UNAM-IFAI.

8 La Ley Federal de Datos Personales en Posesión de Particulares también amplió sustancialmente las facultades, atribuciones y responsabilidades del IFAI, el que para efecto de esta materia se constituye como una autoridad administrativa federal. Asimismo, modificó su nombre al de "Instituto Federal del Acceso a la Información y Protección de Datos Personales" 
febrero de $2014^{9}$, que reforma y adiciona diversas disposiciones de la Constitución Política de los Estados Unidos Mexicanos, en materia de transparencia, a partir de la cual el Artículo $6^{\circ}$ provee de autonomía constitucional al Instituto Federal de Acceso a la Información y Protección de Datos.

\section{Evolución en los estados de la república. La constitucionalización de un derecho fundamental}

El fenómeno de la transparencia no fue ajeno al ámbito local. Cada Estado tiene su ley y su propio instituto, con reglamentos, procedimientos similares, con mayores o menores alcances para el ejercicio del derecho. El sistema federal del Estado mexicano se constituye por 3 poderes federales, 31 entidades federativas y un Distrito Federal y amplía la diversidad de leyes que regulan en los estados y la Federación el derecho de acceso. De esta manera podemos observar la constitucionalización del derecho según el siguiente esquema:

TABLA 1

\begin{tabular}{|c|c|c|c|c|c|c|}
\hline No. & Estado & $\begin{array}{l}\text { Cumplimiento de acuerdo al } 1^{\circ} \text { Transitorio de la adición al } \\
\text { segundo párrafo con siete fracciones al Articulo 6o. de la } \\
\text { Constitución Politica de los Estados Unidos Publicado en el } \\
\text { piario Oficial de la Federación el } 20 \text { de julio de } 2007 \text { Mexicanos }\end{array}$ & $\begin{array}{l}\text { No. De } \\
\text { Modif }\end{array}$ & Estado & Cumplimiento extemporáneo & $\begin{array}{l}\text { No. De } \\
\text { Modif }\end{array}$ \\
\hline 1 & Baja Califomia Sur & $\begin{array}{l}\text { (PL) 20/03/2005, (REF) F. Publicación: 30/11/2007 (NL) } \\
\text { 12/03/2010 }\end{array}$ & 3 & Aguascalientes & $\begin{array}{l}\text { (PL) 26/08/2002, (REF) 22/05/2006, (NL) 06/07/2009, (REF) } \\
23 / 11 / 2009\end{array}$ & 4 \\
\hline 2 & Chiapas & (PL) 12/10/2006, (REF) 29/08/2007, 13/08/2008, 29/10/2008 & 4 & Baja Califomia & (PL) 12/08/2005 (NL) 01/10/2010 & 2 \\
\hline 3 & Chihuahua & (PL) $15 / 10 / 2005$, (REF) 05/07/2008, 11/09/2010, 25/09/2010 & 4 & Campeche & (PL) 21/07/2005, (REF) 15/07/2009 & 2 \\
\hline 4 & Coahuila ${ }^{*}$ & $\begin{array}{l}\text { (PL) 04/11/2003 (REF) 17/04/2007 (NL) 02/09/2008 (REF) } \\
26 / 06 / 2009\end{array}$ & 4 & Guerrero & (PL) 14/10/2005, (NL) 15/06/2010 & 2 \\
\hline 5 & Colima & (PL) 01/03/2003 (REF) 17/01/2004, 03/05/2008, 05/07/2008 & 4 & Hidalgo & (PL) 29/12/2006 & 1 \\
\hline 6 & Distrito Federal & $\begin{array}{l}\text { (PL) } 08 / 05 / 2003 \text { (REF) } 31 / 12 / 2003,28 / 10 / 2005,26 / 12 / 2005, \\
31 / 01 / 2006,29 / 05 / 2006,05 / 01 / 2007 \text {, (NL) 28/03/2008, (REF) } \\
13 / 04 / 2009\end{array}$ & 9 & $\begin{array}{l}\text { Estado de } \\
\text { México }\end{array}$ & $\begin{array}{l}\text { (PL) } 30004 / 2004, \text { (REF) 28/12/2004, 29/12/2006, 01/02/2007, } \\
24 / 07 / 2008\end{array}$ & 5 \\
\hline 7 & Durango & (PL) 27/02/2003 (REF) 04/03/2004, (NL) 13/07/2008 & 3 & Querétaro & (PL) 27/09/2002, (REF) 31/03/2007, 30/12/2008, 03:09/2010 & 4 \\
\hline 8 & Guanajuato & (PL) $29 / 07 / 2003$, (REF) $19 / 05 / 2006,10 / 06 / 2008$ & 3 & Sinaloa & (PL) 26/04/2002, (REF) 20/08/2008 & 2 \\
\hline 9 & Jalisco & $\begin{array}{l}\text { (PL) 22/01/2002, (REF) 06/01/2005, (NL) 17/07/2007, (REF) } \\
12 / 06 / 2008\end{array}$ & 4 & Tamaulipas & (PL) $05 / 07 / 2007$, (REF) 04/11/2009 & 2 \\
\hline 10 & Michoacán & (PL) $28 / 08 / 2002$, (REF) $21 / 11 / 2007,07 / 11 / 2008$ (NL) $16 / 07 / 2009$ & 4 & Thaxcala & (PL) 12/01/2007, (REF) 22/05/2007, 17/12/2009, 26/11/2010 & 4 \\
\hline 11 & Morelos & $\begin{array}{l}\text { (PL) 27/08/2003, (REF) 25/04/2007, 30/04/2008, 05/11/2008, } \\
24 / 12 / 2008,21 / 05 / 2009\end{array}$ & 6 & Yucatán & (PL) 31/05/2004, (REF) 18/08/2008 & 2 \\
\hline 12 & Nayarit & (PL) 16/06/2004, 22/12/2007, (NL) 25/03/2008, 29/05/2010 & 4 & Zacatecas & (PL) 14/07/2004 (REF) 30/08/2008 & 2 \\
\hline 13 & Nuevo Leán & $\begin{array}{l}\text { (PL) 21/02/2003, (REF) 14/07/2004, 19/11/2004, 25/101/2006, } \\
\text { (PLO1/2006, 07/02/2007, 19/07/2008, (NL) 30109/2009 }\end{array}$ & 8 & Federación & (PL) 11/06/2002, (REF) 11/05/2004, 06/06/2006, 05/07/2010" & 4 \\
\hline 14 & Daxaca & (PL) 16/09/2006, 06/10/2007, (NL) 15/103/2008 & 3 & TOTAL DE MO & DDIFICACIONES & 36 \\
\hline 15 & Puebla & (PL) $16,08 / 2004$ (REF) $18 / 07 / 2008$ & 2 & & & \\
\hline
\end{tabular}

16 Quintana Roo $\quad$ (PL) 31/05/2004, (REF) 18/03/2008, 02/07/2008, 30106/2009

17 San Luis Potosí (PL) 20/03/2003, (REF) 18/10/2003, 20/04/2004, 18/10/2007, (NL)

\begin{tabular}{l|ll}
17 & San Luis Potosi & $18 / 04 / 2008,06 / 06 / 2008$ \\
\hline 18 Sonora & (PL) 25/02/2005, (REF) 29/12/2005, 14/08/2007
\end{tabular}

19 Tabasco

20 Veracruz

\begin{tabular}{ll}
19 Tabasco & (PL) 10/02/2007, (REF) 26/12/2007 \\
\hline 20 Veracruz & (PL) 08/06/2004, (NL) 27/02/2007, (REF) 27/06/2008 \\
OTAL DE MODIFICACIOONES
\end{tabular}

Fuente: Elaboración del autor con información contenida en la página web http://www2.scjn.gob.mx/Accesolnformación/default.htm.

De acuerdo con el Artículo 6, las legislaciones de los estados mexicanos reflejan ${ }^{10}$, en todo o en parte, los principios normativos de la Carta Magna, a saber:

1) Principio de máxima publicidad. Todas las leyes reconocen el principio de máxima publicidad o apertura de la información; asimismo, contienen un capítulo de información de acceso restringido.

2) Ausencia de acreditación de interés jurídico. Ninguna legislación establece la obligación de acreditar interés jurídico al solicitante salvo en Querétaro, en dónde el propio órgano garante requiere identificación oficial y número de ésta para presentar su solicitud de acceso.

3) Procedimientos expeditos y órganos garantes especializados. 23 legislaciones (70\%) establecen expresamente el principio de expeditud respecto de los mecanismos de acceso a la información y procedimientos de revisión.

4) Respecto a la autonomía: 27 órganos garantes de los estados (82\%) cuentan con un organismo autónomo, de los cuales 13 (48\%) tienen este carácter por disposición constitucional y 14 (52\%) por Ley.

5) Archivos y publicación de gestión. Respecto a la obligación de publicar los indicadores de gestión, únicamen-

9 Asimismo, el reglamento para la IFAl como organismo autónomo fue publicado el 20 de febrero de 2014, disponible en http://www. dof.gob.mx/nota detalle.php?codigo $=5333316 \&$ fecha $=20 / 02 / 2014$

10 Para un estudio detallado sobre el derecho de acceso a la información pública en los estados mexicanos ver Ford Guerra, Oscar M. (2011). Los Órganos Garantes de Transparencia. Integración y características. México: UNAM. Instituto de Investigaciones Jurídicas de la Universidad Autónoma de México; López Ayllón, Sergio (2010) Métrica de la Transparencia 2010. Recuperado de. http://www.metricadetransparencia.cide.edu/Metrica\%202010.pdf 
te el $64 \%$ de las leyes establece dicha obligación para todos los sujetos obligados.

6) Publicidad del uso de recursos públicos entregados a personas físicas y morales. El $79 \%$ de las leyes cumplen con dicha obligación, de las cuales el $77 \%$ señalan la obligación de manera general.

Así, los avances que se han registrado entre 1998 a 2013 para crear mecanismos de transparencia y de rendición de cuentas se inscriben en tres grandes vertientes: el robustecimiento del control interno cada uno de los tres poderes federales, la neutralización del sistema (los tres poderes empiezan a vigilarse mutuamente) y el avance en la capacidad de la ciudadanía para acceder a la información generada por el gobierno, en concordancia con procesos similares que se replican internacionalmente. No obstante los avances enumerados y como conclusión preliminar, coincidimos con las palabras de Ricardo Cortés Ontiveros (2005), quien subraya que el reto más importante que tiene México en materia de transparencia, consiste en hacer que mediante su ejercicio cívico y responsable, se implante verdaderamente una responsable cultura ciudadana de transparencia, ejercitada por los gobernantes en favor de los gobernados para poder recuperar la confianza del ciudadano-gobernado en autoridades que rinden cuentas ante él. Si bien la rendición de cuentas ha generado gran cantidad de datos y se considera como un factor indispensable para la democracia, todavía no se han hallado los medios para cristalizar su efectividad como así tampoco se observa que la percepción de la transparencia, se aprecie como valor institucional por parte de la ciudadanía, tema que será ampliado más adelante.

\section{El Instituto Federal de Acceso a la Información y Protección de Datos}

Tal como lo señaláramos, 11 la última reforma constitucional de 2014 en su Artículo $6^{\circ}$ provee de autonomía constitucional al Instituto Federal de Acceso a la Información y Protección de Datos (IFAI), constituyéndose en un organismo autónomo con personalidad jurídica y patrimonio propio, con plena capacidad técnica, de gestión, así como para decidir sobre el ejercicio de su presupuesto y determinar su organización interna, responsable de garantizar el cumplimiento del derecho de acceso a la información pública y a la protección de datos personales en posesión de los sujetos obligados en los términos que establezca la ley señalando el inicio de una nueva etapa en el sistema de acceso a la información en el país. El alcance de su misión involucra garantizar a las personas el derecho de acceso a la información pública gubernamental, preservar los datos personales y resolver sobre aquellos casos en los cuales se niegue el acceso a la información. El IFAl interviene a instancia del solicitante, cuando los sujetos obligados (dependencias o entidades de la administración pública federal) no han satisfecho sus obligaciones de proporcionar la información. El medio procesal para remediarlo es un recurso administrativo, el de revisión. En otras palabras, a través del recurso se somete a revisión un acto de la administración ante la misma administración.

\section{El índice de percepción de la corrupción en México (1998-2013)}

La organización internacional Transparencia Internacional publica desde 1995 el índice de percepción de la corrupción que mide, en una escala de cero (percepción de muy corrupto) a diez (percepción de ausencia de corrupción), los niveles de percepción de corrupción en el sector público en un país determinado y consiste en un índice compuesto, que se basa en diversas encuestas a expertos y empresas. Se entiende que un país que posee un IPC de 6 puntos o más, se encuentra en niveles más cercanos a un punto óptimo de corrupción, mientras que aquellos que poseen una puntuación de 4 o menos experimentan niveles de corrupción elevados. En México, el valor medio del IPC desde el año 1998 hasta 2013 arroja un resultado de 3.5 puntos, ubicándose en el grupo de aquellos países con niveles de corrupción más elevado a nivel mundial. A continuación se presentan los resultados del relevamiento:

11 La Ley Federal de Datos Personales en Posesión de Particulares también amplió sustancialmente las facultades, atribuciones y responsabilidades del IFAI, el que para efecto de esta materia se constituye como una autoridad administrativa federal. Asimismo, modificó su nombre al de "Instituto Federal del Acceso a la Información y Protección de Datos Personales" 
TABLA 2. ÍNDICE DE PERCEPCIÓN DE LA CORRUPCIÓN (1998-2013)

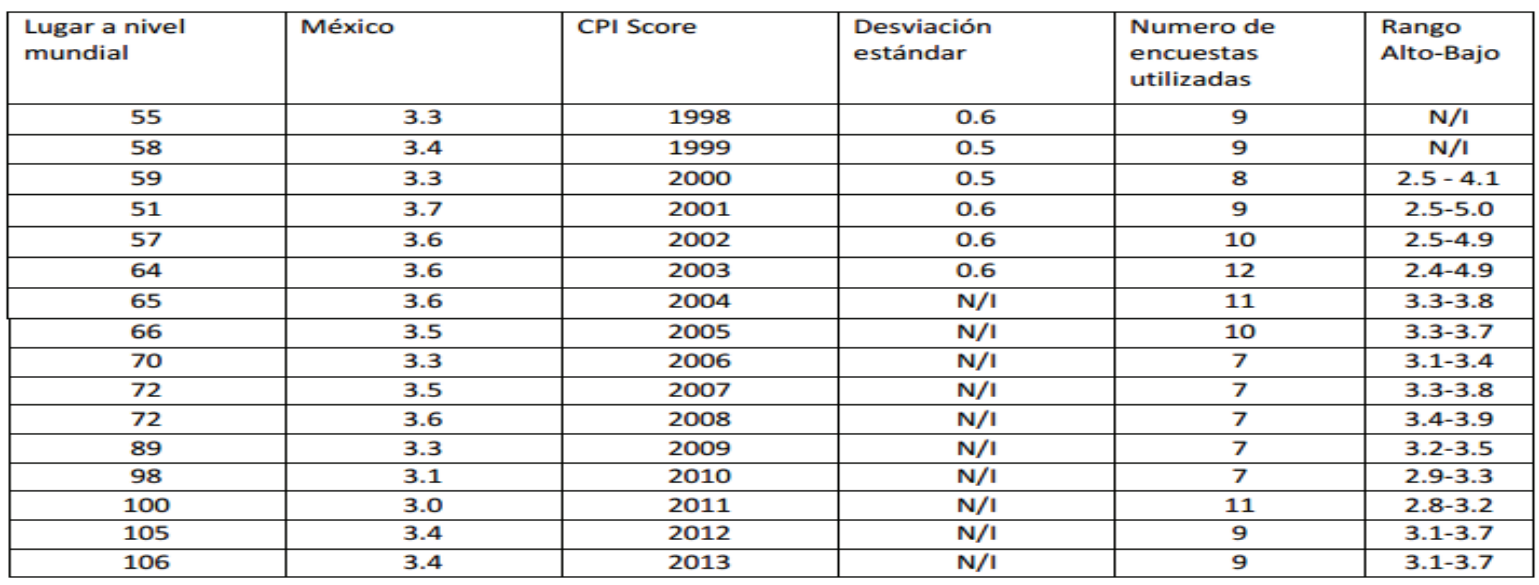

Fuente: IPC Transparency International 1998-2009 12

Los datos señalan que desde el año 1998, el IPC arroja valores constantes de la medición de la corrupción en México, denotándose disminuciones de sólo un decimal respecto del año anterior en determinados años (2005 respecto de 2006; 2009 respecto de 2008; 2011 respecto de 2010). Por su parte, se han registrado aumentos en el IPC respecto del año anterior en los años 1999; 2001, 2007, 2008; 2012 y 2013. Sin embargo, los resultados no reflejan un cambio considerable en la puntuación. En el próximo apartado se procederá a cotejar estas variables juntamente con aquellas normativas para reflexionar sobre la relación establecida entre ambas.

\section{ANÁLISIS DE RESULTADOS}

Luego del análisis de los datos del punto 4.1, consideramos que el sistema normativo mexicano relativo a la transparencia de la información evidencia mecanismos jurídicos e institucionales que permiten a cualquier ciudadano no sólo el acceso a la información sino también a los medios necesarios para realizar los reclamos correspondientes. En materia de transparencia, el escenario actual en México presenta mecanismos favorables para el acceso a la información desde la aprobación de la Ley Federal de Transparencia y Acceso a la Información Pública y la consecuente obligación de las dependencias del gobierno federal a abrir a la sociedad todos sus archivos, documentos e información. Sin embargo, la transparencia de la información pública y el conocimiento de determinados datos no han resultado efectivos para la disminución de la clasificación del país como favorable a perpetrar actos corruptos por parte de sus funcionarios. Sin embargo, el contexto que se vincula a las variables presentadas (en el punto 4.2), se presenta como insatisfactorio en término de resultados de abuso de poder, acuerdos clandestinos y sobornos. Recurrentemente, los informes de TI, y recientemente el Índice de Percepción de la Corrupción 2013, reiteran que México se encuentra entre los países más corruptos. Para dicho año, el país se ubicó en la posición 106 de 177 a nivel mundial con 34 puntos, la misma que obtuvo en 2012 coincidiendo en la misma tendencia en índices en mayor o menor medida iguales en los años anteriores. Tales niveles indican una alta proliferación de prácticas corruptas y se ha identificado que la población mayoritaria señala que las acciones emprendidas por el Presidente en turno en contra de la corrupción son "pocas" o "regulares". En México los alcances y límites del control externo e interno a los cuales se encuentra expuesto el Poder Ejecutivo son frágiles (Guerrero Gutiérrez, 2003); "ni los índices del IPC (por el lado de la demanda de corrupción) ni los del BPI (fuentes u oferta de sobornos) referidos anteriormente muestran evidencias perceptibles de mejoras desde que se han estado publicando periódicamente" (Scholoss, 2013) además, los indicadores de percepción de la corrupción indican que ha habido muy pocos avances en su combate (salvo una mayor concientización sobre el tema), no obstante el número creciente de convenciones, convenios internacionales, aprobación de nuevas legislaciones, etc. Según explica Morris (1991), el fenómeno de la corrupción extendida y su proliferación en la vida civil obedece a "la glorificación (sic) cultural de la corrupción en ciertos sectores de la población, por el surgimiento de una moralidad distorsionada en la clase media, por la desviación de la responsabilidad individual y por la difusión de la desconfianza y el cinismo hacia el gobierno y los funcionarios públicos". Se estima que las empresas mexicanas pagan cada año 8.400 millones

12 La columna puntuación de CPI de 1998 se refiere a las percepciones del grado en que la corrupción es vista por empresarios, en donde un 10.00 perfecto sería un país totalmente libre de corrupción. 
de dólares en sobornos a legisladores y otros funcionarios públicos para influir en el diseño y la aplicación de las leyes o la financiación de campañas electorales. Asimismo, entre Latinoamérica, su situación como país no es más favorable. Si existen entonces mecanismos normativos e institucionales que permiten la participación ciudadana y la transparencia de los actos de gobierno, ¿por qué dichos mecanismos no reflejan una disminución en los índices de corrupción? ¿Qué otros factores se pudieran presentar como complementarios a la relación transparenciacorrupción en México por los que no se ve impactada positivamente por la aparición de normas jurídicas que han sido generadas para incentivar la participación ciudadana en los actos públicos?

Respondiendo a la primer interrogante, concordamos con los argumentos de la literatura revisada (en el apartado 2), la cual indica que los cambios legislativos no se traducen de forma inmediata o automática en un impacto en el fenómeno de la corrupción de un país. Coincidiendo con Arellano Gault (2000) debemos desctacar que una reforma jurídica como política organizativa de la transparencia no se traduce inmediatamente en el hardware organizativo del Estado, debido a las tensiones múltiples que la relación genera. Así, la relación transparenciacorrupción debiera ser interpretada como un engranaje complejo de relaciones entre los grupos y los individuos, racionalizado y hecho razonablemente en conjunto de valores y de normas de la acción organizativa.

Por otra parte, y sin desconocer los avances enumerados en materia normativa, coincidimos con las palabras de Ricardo Cortés Ontiveros (2005), quien subraya que el reto más importante que tiene México en materia de transparencia, consiste en hacer que mediante un ejercicio cívico y responsable, se implante verdaderamente una responsable cultura ciudadana de transparencia, ejercitada por los gobernantes en favor de los gobernados para poder recuperar la confianza del ciudadano-gobernado en autoridades que rinden cuentas ante él. Si bien la rendición de cuentas ha generado gran cantidad de datos y se considera como un factor indispensable para la democracia, todavía no se han hallado los medios para cristalizar su efectividad una fuerte cultura por la transparencia por parte de la ciudadanía.

Existen diferentes factores para explicar lo antedicho. En primer lugar, puede obedecer a un complejo abordaje jurídico de la política a llevar a cabo, lo cual se verá reflejado en un exceso de aspectos formales, que por lo general son lentos, burocráticos y de mucha menor flexibilidad que la que se requiere para hacer frente a la destreza, versatilidad e imaginación de los agentes que practican la corrupción (Schloss, 2013). En segundo lugar, cuestiones de carácter sociológico también imponen ciertas limitaciones, por ejemplo, la falta de apropiación de los mecanismos por parte de la sociedad pueden considerarse como un fenómeno negativo al desarrollo de legitimidad del derecho de acceso y reclamo de respuestas. A su vez, los mecanismos de control conllevan a percibirlos como complejos para el procesamiento de sus resultados a nivel individual e institucional. De igual manera, la escasez de entidades independientes de confianza puede ser vista como un condicionamiento para el desarrollo de una política ciudadana participativa. Finalmente, una percepción ética y moral negativa de la función pública actúa como elemento revelador del funcionamiento del Estado (Guerrero Gutiérrez, 2013). Tal como señala Prats Català (2007) la falta de valores y su vinculación con la corrupción causan el deterioro de la credibilidad y la imagen de lo público, recayendo sobre ella la sospecha de dar cobijo a conductas poco lícitas o de subyacentes intereses espurios alejados del valor público. De lo antedicho surge entonces indagar en respuestas a nuestra segunda interrogante, a saber qué otros factores podemos proponer como complementarios a la relación transparencia-corrupción para impactar positivamente sobre la segunda variable de la relación.

En este sentido consideramos que la misma debe ir acompañada de otros elementos favorables para un efectivo impacto de las acciones de publicidad y de rendición de cuentas, como el desarrollo y la capacitación de la sociedad civil; el fortalecimiento de la administración pública; el establecimiento de un combate frontal a la corrupción y mecanismos de intermediación eficaces. Esto implica establecer marcos específicos de comparación y medición de las actividades organizativas, con el fin de establecer parámetros más o menos medibles que permitan a los actores organizacionales calcular los costos y beneficios de su interacción, así como compartir el sentido de la acción y la posibilidad de construir nuevos valores que permitan dar sentido a la transparencia de una forma internalizada y no sólo como respuesta a un castigo o a una amenaza. Para que lo último se cristalice sería necesario no sólo que el gobierno que sea capaz de responder a las necesidades de sus ciudadanos en términos de legitimidad sino que también exista una identificación y definición de las necesidades de los usuarios en comunicación y consulta con los mismos, respecto de una evaluación del diseño de las propias políticas y estructuras que promueven la transparencia como así también de la idoneidad de la organización que las implementa y los resultados de la acción pública, a fin de poder examinar con detenimiento "la relación causal que contiene el diseño de la política pública, el tipo de organización pública que es responsable de su implementación y el modo de gobernanza o proceso de gobernar ya que la contingencia de la organización, puede o no asegurar la implementación y gestión eficaz de las políticas, programas y servicios" (Aguilar, 2010). 
Analizar la gestión de políticas orientadas a la reducción de la corrupción únicamente desde un enfoque gubernamentalista, considerando que un acto corrupto obedece a un crimen de cálculo y oportunidad sin ninguna relación con otros factores, implicaría incurrir en un reduccionismo que no termina de responder a nuestro interrogante inicial, es decir, la necesidad de identificar otras herramientas para incidir positivamente en un resultado concreto que reduzca los índices de corrupción. El enfoque gubernamentalista sostiene que la idea de que el gobierno es suficiente en la solución de los problemas sociales y en la producción de los objetivos sociales preferidos, por lo que tanto el éxito como el fracaso relativo es atribuible exclusivamente al gobierno y sus organismos. Por lo tanto, sostenemos que el tratamiento de estos problemas requiere tratar las causas económicas, políticas e institucionales subyacentes y, sobre todo, culturales. Es decir, existen acciones que, en conjunción con las ya existentes (leyes de acceso a la información y mecanismos de rendición de cuentas) pueden ser viables para un impacto positivo en la evaluación de la gobernanza y disminución de la corrupción del Estado.

Por ello, si bien la democracia electoral es una condición necesaria más no suficiente para producir gobiernos eficaces y que rindan cuentas, resulta necesario construir o fortalecer instituciones de rendición de cuentas que obliguen a los gobernantes a someterse al veredicto y control de otros poderes de gobierno y al veredicto de los ciudadanos, más allá de la celebración de elecciones. México ha concluido con relativo éxito su proceso de transición a la democracia pero lo que se le exige ahora a sus gobiernos es que presenten resultados por medio del afianzamiento de instituciones de rendición de cuentas sujetas a evaluación, tanto en el ámbito federal como en los gobiernos estatales y municipales. De otra forma, la democracia mexicana puede convertirse en un concepto vacío al no producir gobiernos eficaces y honestos.

Existen varios vehículos para evaluar las áreas concretas de vulnerabilidad como son las encuestas sistemáticas para "triangular" y evaluar cada una de las áreas de gobierno vulnerables a la corrupción o ciertas medidas dentro del modelo de gobernanza del Estado y de la sociedad civil, como es el "entender y diagnosticar cómo funciona el sistema corrupto específicamente en el contexto particular del país o región; superar la resistencia política y burocrática, y generar apoyo de los que puedan generar cambios; y crear un plan de acción secuencial para superar los sistemas corruptos, romper la cultura del cinismo y generar ímpetu político y de transformación de gobierno, sociedad civil y sectores empresariales"(Schloss, 2013). Un modelo de gobernanza' y su consecuente evaluación se encontraría entonces orientado a incluir otros factores y variables tanto intra estatales como societales, sustentando la conducción política y democrática del Estado, con estabilidad y cohesión, pero desde la perspectiva de la base social o de la ciudadanía, en donde la misma pueda encontrar canales de expresión, participación y apropiación de la información pública, y en donde la rendición de cuentas, la transparencia, la fiscalización y el acceso a la información pública se encuentre orientado más a los intereses, la participación y el control ciudadano. A pesar de la existencia de un sistema de pesos y contrapesos todavía subsisten causas, defectos e incentivos que pueden propiciar infracciones a las normas. También se estima necesario repensar los alcances y límites del control interno o autocontrol del Poder Ejecutivo para avanzar hacia un auténtico equilibrio entre poderes en México, ya que todavía los controles externos a los que está expuesto el Ejecutivo son todavía frágiles e insuficientes ${ }^{14}$.

Estimamos que para crear una estructura acompañada de incentivos para que los funcionarios públicos y los propios ciudadanos se involucren en las acciones correctivas, primeramente es necesario comprender las causas y los costos que los actos de corrupción traen consigo. Seguidamente, debieran trazarse metas a corto, mediano y largo plazo que prevean: a) la selección de los agentes de cambio; b) la determinación de los incentivos, recompensas y penalizaciones; c) medición de los resultados; d) y la reestructuración de la relación entre los actores principales, los agentes de cambio y los beneficiarios: reducir el monopolio, clarificar y limitar la discreción y mejorar la rendición de cuentas (Schloss, 2013).

Por último y con relación al factor social como variable incidente en la configuración de políticas de transparencia, los itinerarios del proceso general de modernización por las teorías funcionalistas sostienen que la modernización social deviene luego de una modernización política económica. Si bien dicho abordaje consideraba

13 Cabe distinguirlo del término gobernabilidad entendida como la estabilidad y orientación de la conducción política del Estado, preservando la estabilidad y la cohesión, pero desde la perspectiva del propio Estado. Según este enfoque, la gobernabilidad considera que el problema directivo es suficientemente resuelto si se dota o se provee de nuevo al gobierno con las capacidades requeridas (instituciones, fiscales, administrativas, coactivas...) para estar en aptitud de dirigir a la sociedad.

14 La identificación de los problemas no siempre conlleva a métodos sofisticados: en las etapas iniciales de la acción correctiva una estrategia más intuitiva es muchas veces suficiente para por lo menos detectar las áreas “más obvias" de disfunción. Ciudadanos, empresarios y funcionarios públicos que trabajan en instituciones sistemáticamente corruptas pueden analizar dónde y cómo se posiciona la corrupción. Esto puede ser sorprendente, pero muchas veces este es el caso, con tal que el enfoque sea orientado hacia el sistema corrupto y no a los individuos. 
al factor social como residual para la modernización, hoy en día tal factor muestra un dinamismo que introduce sus propias condiciones, prioridades y ritmos y que por consiguiente, debe ser considerado. No son pocas las evidencias que indican el desajuste en el movimiento permanente de las tres dimensiones y una exigencia de coordinación sobre la base de una marcada diferenciación de expectativas y lógicas de operación. Las sociedades modernas, debido al alto grado de especialización funcional, son sociedades sin centro, sin estructura central capaz de regular enteramente las relaciones intrasistémicas y sociales, lo cual hace cada vez más difícil la posibilidad de ordenar coherentemente las funciones de cada sistema parcial en atención a una lógica general de la sociedad y a un vértice constituido para darle dirección a tal lógica. Es por ello que estimamos que la coordinación de políticas anticorrupción, sin la debida integración sistémica no puede brindar resultados favorables.

\section{CONCLUSIONES}

El presente artículo tuvo como fin presentar un estudio de caso que permita corroborar aspectos ya expuestos por otros autores respecto de la relación entre transparencia y corrupción analizando la relación entre ambos factores como se presenta en México. Hemos argumentado que los cambios normativos que facilitan el acceso a la información no han sido suficientes para influenciar positivamente en la relación entre transparencia y corrupción. Una explicación posible se ha fundado en el argumento que sostiene que las reformas normativas para poder ser evaluadas, deben ser analizadas en el largo plazo. Sin embargo, hemos sostenido que aún si lo último fuera cierto, y en concordancia con la literatura que ha estudiado la compleja relación transparencia-corrupción, en México debieran tomarse en consideración otros factores extra-jurídicos para contribuir positivamente a la disminución de los índices de corrupción. Si bien no desconocemos la importancia de la existencia de normas jurídicas que faciliten la transparencia en su relación con la corrupción, hemos explicado las razones por las cuales en el caso Mexicano este vínculo carece de mayor desarrollo de factores complementarios tanto de carácter institucional como social y cultural.

Así, si bien el acceso a la información pública se encuentra orientada a la transparencia y a la participación ciudadana, dichos fines no implican necesariamente el diseño de sistemas y procesos para transformar el comportamiento de la administración pública y que los mismos necesiten de otras acciones para poder obtener los resultados esperados. Si los resultados arrojados por los diferentes índices que miden el fenómeno de la corrupción en México no reflejan tal correspondencia puede afirmarse que los valores de eficacia, eficiencia y receptividad promovidos por las leyes y políticas de transparencia no configuran, por sí solos o necesariamente, un impacto positivo en la reducción de la corrupción y en consecuencia, dar bases a un gobierno ético y responsable.

Se considera que la visión del potencial de la transparencia como instrumento para la mejora de las instituciones políticas y sociales no debe hacer referencia a políticas públicas aisladas sino, más bien, a diferentes partes de un abordaje sistémico del problema de la corrupción. Por ello sugerimos que si se tiene como fin el sustentar un modelo de gobernanza y un método de su evaluación con éxito, además de la legitimidad institucional, el cumplimiento de la legalidad y la consecución de resultados, se deben añadir -y por lo tanto definir- por una parte, la capacidad directiva de los gobiernos como valor de referencia fundamental en el ejercicio de la evaluación de la acción pública como así también generar una cultura de la participación por parte del cuerpo ciudadano.

Cualquier propuesta vertida para obtener los efectos buscados no debe dejar de lado los factores culturales, puesto que, en definitiva, tanto dentro como fuera de las instituciones públicas, son los seres humanos quienes conducen los grupos sociales. Se trata de evidenciar los antagonismos relacionados con la cultura que el país debe superar a fin de consolidar, desde afuera hacia adentro y desde abajo hacia arriba, un dilema cultural que en muchos casos se encuentra en vías de transformación pero que en otros todavía no ha despertado el cambio. Estas se puede sintetizar en cuatro grandes disyuntivas, a saber: a) cultura del secreto vs. cultura de la rendición de cuentas: la cual implica involucramiento y reclamos por parte de los destinatarios interesados a fin de lograr el efecto buscado; b) cultura de la votación vs. cultura de la participación: es decir, superar la visión según la cual la participación en la democracia por parte del ciudadano equivale solamente a la votación en las elecciones y la percepción de la ciudadanía que estima la existencia de una baja probabilidad de que su participación en la vida cívica produzca un cambio de comportamiento en el sector administrativo; c) cultura de la ignorancia de la cosa pública vs. cultura del conocimiento, lo que indica que si las necesidades desean verse traducidas en demandas y tener la debida satisfacción, el actor debe estar en conocimiento de una serie de procedimientos, modalidades de acción y rituales propios de la acción político-institucional y lograr apropiarlos; d) cultura individual vs. cultura colectiva, según la Encuesta Mundial de Valores organizada por la Universidad de Michigan, la confianza interpersonal está estrechamente vinculada con la democracia, con el respeto a la ley y con el asociacionismo, es decir, que la buena 
administración no es nada concedido por la gracia de los gobernantes sino un derecho que va conquistándose por la ciudadanía activa y organizada.

Como conclusión final, estimamos que en México toda política de desarrollo que promueva reformas institucionales centradas únicamente en el aumento de la transparencia resulta insuficiente para el fin que se predispone a combatir. Desde una visión integradora y sistémica, la misma debe ir acompañada de medidas para fortalecer la capacidad de los pueblos de actuar sobre la información disponible. Si deseamos que la transparencia funcione al servicio de la lucha contra la corrupción la mera detección de los abusos no basta. Un sistema también necesita autoridades con la capacidad y mando para actuar según la información y denuncias recibidas. En ausencia de tal sistema, las medidas resolutivas respecto de las denuncias junto a un nivel limitado de sanciones pueden allanar el camino para un mayor fraude. La participación se erige en una suerte de contralor peculiar: en la medida en que el gobierno de turno no satisface las demandas sociales de transparencia, pierde legitimidad automáticamente. Es aquí donde la incidencia del poder del ciudadano como actor político cobra singularidad y presencia necesaria para la solución del problema planteado y para alcanzar políticas públicas de saneamiento institucional verdaderamente efectivas.

\section{BIBLIOGRAFÍA}

Aguilar, L. y Bustelo, M. (Julio 2010). Gobernanza y evaluación: una relación potencialmente fructífera en Gestión y Análisis de Políticas Públicas. Instituto Nacional de Administración Pública, 3-10.

Álvaro Cuervo Cazurra "Transparency and corruption" en el libro Forssbaeck, J. and Oxelheim, L. (2014): The Oxford Handbook of Economic and Institutional Transparency, Oxford: Oxford University Press. DOI: 10.1093/oxfordhb/9780199917693.013.0015.

Arellano, D. (2000). Reformando al gobierno: una visión organizacional del cambio gubernamental. México: Porrúa.

Arellano Gault, D. (Septiembre 2007). Fallas de transparencia: hacia una incorporación efectiva de poíticas de transparencia en las organizaciones públicas. Revista de Ciencias Sociales Convergencia, Número 45, 31-45.

Bauhr, M. and Grimes, M. (Abril, 2014), Indignation or Resignation: The Implications of Transparency for Societal Accountability. Governance Vol 27, 291-320, DOI http://dx.doi.org/ 10.1111/gove.12033

Bellver, A. (Junio 2007). Reformas en materia de transparencia: segunda generación de cambio institucional. Revista del CLAD Reforma y Democracia, Volumen 38.

Molina, E. y Vieyra, J. (Noviembre 2012). La transparencia como herramienta de política pública en Gobierno abierto y transparencia focalizada. Banco Interamericano de Desarrollo. 4-59. DOI http://dx.doi.org/IDB-MG-137

Della Porta, D. y Vanucci, A. (2002). Los recursos de la corrupción: algunas reflexiones sobre el caso italiano en Zona abierta (98-99), 85-118.

Doyle, K. (julio 2003). Comentarios sobre la Ley Federal de Transparencia y Acceso a la Información Pública Gubernamental. Derecho Comparado de la Información, (2), 163-172.

Florini, A. (1999), Does the Invisible Hand Need a Transparency Glove? The Politics of Transparency. Paper prepared for the Annual World Bank Conference on Development Economics, Washington, D.C., April 28-30.

Graham, M. (2002). Democracy by Disclosure, the Rise of Technopopulism. Washington D.C.: Brookings Institution Press. DOI: 10.1002/pam.10168.

Guerrero, E. (Agosto 2003) Cultura de transparencia y rendición de cuentas en la gestión pública en Ensayos, Cultura de transparencia y rendición de cuentas en la gestión pública. Instituto Federal Electoral, 11-26.

Lindstedt, C. y Naurin D. (Junio 2010). Transparency is not Enough: Making Transparency Effective in Reducing Corruption. International Political Science Review, volume 31 (3), 301-322. DOI: 10.1177/0192512110377602.

Morris, S. (1991). Corrupción y Política en el México Contemporáneo. México: Ed. Siglo XXI editores.

Nassens, H. (2010). Ética Pública y Transparencia. Artículo presentado en el Congreso Internacional 1810-2010: 200 años de Iberoamérica (Septiembre 2010, España). Consejo Español de Estudios Iberoamericanos, Cursos e Congresos, 2113-2130. 